\title{
Tumorgenetik
}

\section{Karzinogenese: Big Bang oder kontinuierliche Evolution?}

KERSTIN HAASE, ROLAND F. SCHWARZ

MAX-DELBRÜCK-CENTRUM FÜR MOLEKULARE MEDIZIN, BERLIN

\section{Intra-tumour heterogeneity is a key characteristic of tumours and} poses significant clinical challenges. Despite extensive research, the evolutionary processes shaping cancer genomes are not yet fully understood. We here discuss two conflicting theories about the evolution of solid tumours: the Big Bang model and the classical model of continuous evolution. Using chromosomal instability we shed light on the preferred mode of cancer evolution and its implications for personalised treatment.

DOI: $10.1007 / \mathrm{s} 12268-020-1488-\mathrm{x}$

(C) Die Autoren 2020

Tumorheterogenität und Krebsevolution haben sich in den letzten zehn Jahren zu Schlüsselthemen der translationalen Krebsforschung entwickelt. Peter Nowell war einer der Wegbereiter dieser Theorie, die er 1976 in „The clonal evolution of tumour cell populations“ [1] darlegte. Jüngste Studien wie „The Cancer Genome Atlas“ und das „International Cancer Genome Consortium“ rückten die Theorie erneut in den Fokus und haben unser Verständnis über Krebsevolution und Tumorheterogenität entscheidend geprägt und den Weg zur Entwicklung neuer Medikamente für die zielgerichtete Krebstherapie bereitet.

Diese Studien zeigten im Detail intratumorale Heterogenität (ITH) auf, die Koexistenz genetisch unterschiedlicher Subklone innerhalb eines Tumors. ITH ist klinisch höchst relevant, da sie hauptverantwortlich ist für die Resistenzentwicklung gegen klassische Chemotherapeutika, aber auch insbesondere gegen Verfahren der zielgerichteten Krebstherapie [2]. Eine zufällig akquirierte Resis-

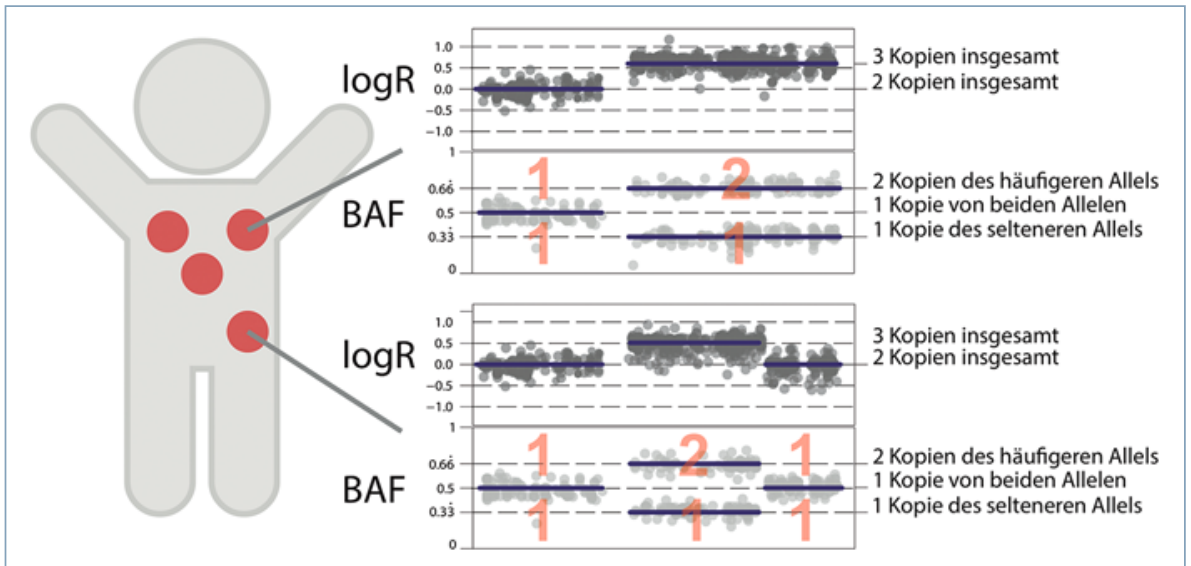

$\Delta$ Abb. 1: Detektion von SCNAs aus Sequenzierdaten. Die Anzahl der Sequenzierreads an beiden Allelen heterozygoter Varianten erlaubt die Bestimmung der Gesamt-DNA-Anzahl aus dem logR und die Verteilung der Kopien über beide elterliche Allele durch die BAF. In Regionen mit SCNA teilt sich die BAF-Bande in zwei Banden, abhängig von der Haplotypstruktur des Patienten. BAF: B-Allel-Frequenz; logR: Gesamtanzahl Kopien; SCNA: somatic copy number alteration. tenzmutation in einer Zelle kann genügen, um nach erfolgreicher therapeutischer Auslöschung der Mehrheit der anderen Klone einen schnellen resistenten Rückfall herbeizuführen. Diese Prozesse im Detail zu verstehen ist das erklärte Ziel der Krebsforschung.

\section{Kopienzahlaberrationen als evolutionäre Marker}

Chromosomale Instabilität (CIN), eine Schlüsseleigenschaft vieler Tumore, ist dabei von besonderem Interesse. CIN beschreibt die Befähigung eines Tumors, sein Genom zu rearrangieren und auch großflächige Änderungen am Karyotyp in der Form von somatischen Kopienzahlaberrationen (somatic copy-number alteration, SCNA) zu tolerieren. Da die Sequenzierung einzelner Proben eines Tumors oft nicht ausreicht, um das gesamte Ausmaß der ITH zu erfassen, bedient sich das Feld zur Quantifizierung von CIN oft so genannter Multiregions-Studien, bei der der chirurgisch entfernte Tumor in mehrere Regionen unterteilt und anschließend sequenziert wird [3].

Dabei werden zur Detektion von SCNAs heterozygote Keimbahnvarianten genutzt, um reads einem der beiden elterlichen Allele zuzuweisen (Abb. 1). Zwei Kenngrößen ermöglichen dann die Detektion von SCNAs: 1. das logarithmische Verhältnis der Sequenziertiefe im Tumor zu der im Normalgewebe als Maß für die Gesamtanzahl an Kopien $(\log R)$ und

2. das Verhältnis der Anzahl der Sequenzierfragmente der beiden elterlichen Alle-

le zueinander, die B-Allel-Frequenz (BAF). In einem diploiden Teil des Genoms bleibt das $\log$ R bei einem Wert von Null und die BAF liegt bei 0,5 , da die Hälfte der reads zufällig vom mütterlichen und die andere Hälfte vom väterlichen Allel stammen. Im Fall einer SCNA erhöht oder erniedrigt sich der logR-Wert und die BAF zeigt eine allelische Imbalance an (Abb. 1). Die Ermittlung allelspezifischer SCNAs ermöglicht somit auch die Detektion der klinisch hoch relevanten kopienzahlneutralen Heterogenitätsverluste. 

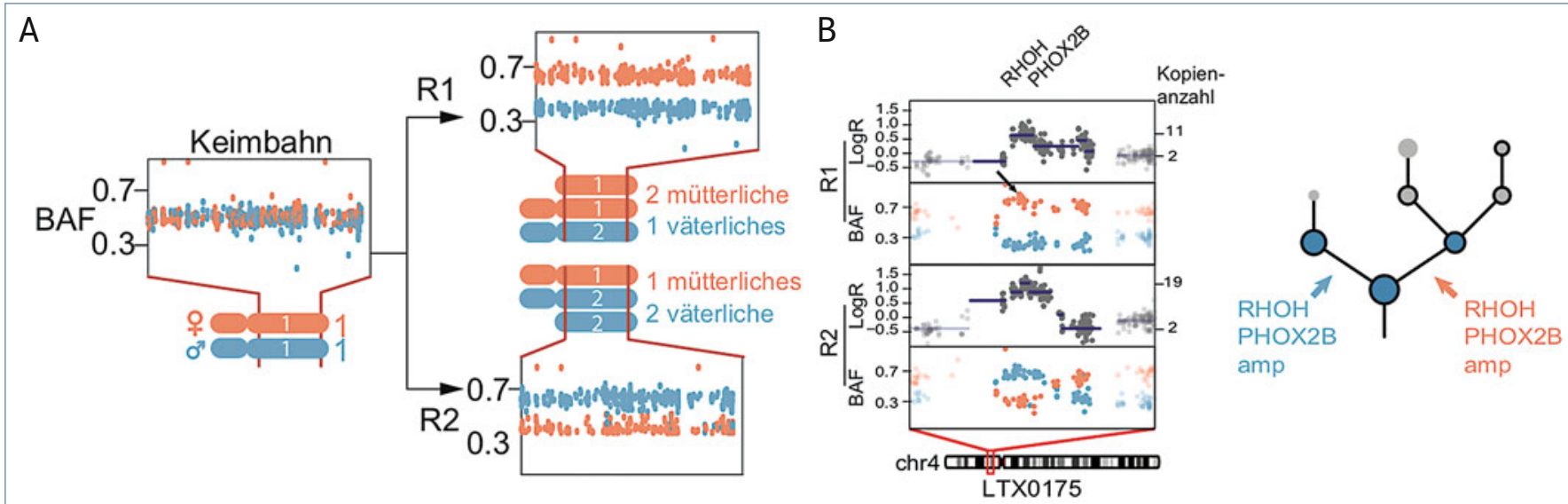

A Abb. 2: Multi-Regions-Referenz-Phasing (MRP) und mirrored subclonal allelic imbalance (MSAI). A, MRP erlaubt die Zuweisung von SCNAs zu elterlichen Haplotypen in Multi-Regionsstudien. So können Ereignisse unterschieden werden, die in unterschiedlichen Regionen des Tumors auf unterschiedlichen Haplotypen auftreten (MSAI). B, MSAI-Ereignis in Lungenkrebs: Eine fokale Amplifikation des PHOX2B/RHOH-Lokus erzeugt > 10 Kopien. Die Amplifikation fand parallel in zwei Regionen des Tumors auf unterschiedlichen Haplotypen (orange und blau) statt. Abbildung aus [10].

Um der Herausforderung der Rekonstruktion von Krebsevolution aus SCNA-Profilen zu begegnen, entwickelten wir MEDICC [4], einen Algorithmus zum paarweisen Vergleich genomischer SCNA-Profile und zur Rekonstruktion evolutionärer Bäume mittels einer Distanz der minimalen Ereignisse beliebiger Länge (minimum event distance). Mittels MEDICC waren wir in der Lage, räumliche und temporale ITH in Eierstockkrebs aufzuklären und einen direkten Zusammenhang zwischen ITH und einem schlechteren klinischen Verlauf herzustellen [5].

Durch die Integration von SCNA-Profilen und Punktmutationen lässt sich zudem die relative Zeit in der Tumorevolution ermitteln, zu der eine SCNA erfolgt ist. Je mehr Mutationen auf zwei oder mehr Kopien einer Region vorliegen, desto später ist die SCNA erfolgt, da sich viele Mutationen bereits davor akkumulieren konnten. Mit dieser grundlegenden Theorie konnten wir die evolutionäre Entwicklung von verschiedenen Tumortypen nachvollziehen [6].

\section{Der Big Bang der Krebsevolution}

Das Aufkommen der Multiregions-Studien erlaubte detaillierte Einblicke in die Evolution vieler Tumortypen. Eine entscheidende Diskussion entstand bezüglich der Dynamik der evolutionären Veränderungen. A. Sottoriva et al. entwickelten 2015 das Big BangModell der Krebsevolution aus Beobachtungen in Kolonkarzinomen [7]. Demnach sollten die meisten detektierbaren Mutationen und der stärkste Selektionsdruck sehr früh in der Karzinogenese erfolgen. Spätere Mutationen sind demnach aufgrund der expandie- renden Tumormasse nur schwer detektierbar und erreichen selten Fixation in der Zellpopulation. Die Hypothese erregte Aufsehen und das Feld spaltete sich in zwei Lager, solche die das Big Bang-Modell für glaubhaft hielten und solche, die kontinuierliche Evolution und Selektion als das dominierende Prinzip der Krebsevolution hielten.

Um zur Beantwortung dieser Frage beizutragen, entwickelten wir 2015 einen neuen Algorithmus zur eindeutigen Zuweisung allelspezifischer SCNAs zu elterlichen Haplotypen. Bisherige Algorithmen waren zwar in der Lage, an jeder heterozygoten Keimbahnvariante die Kopienzahlen beider Allele mit einer gewissen Genauigkeit zu bestimmen [8], es war jedoch unklar, ob zwei SCNAs an unterschiedlicher Position in einem Chromosom auf der selben oder auf unterschiedlichen elterlichen Kopien des Chromosoms auftraten. Dieses Problem ist, analog zu der verwandten Fragestellung in der Genetik, als phasing problem bekannt. Hier bieten Multiregions-Datensätze einen besonderen Vorteil: Da die Phase der Keimbahnvarianten zwar zunächst unbekannt, aber in allen Regionen des Tumors konstant ist, kann durch direkten Vergleich der BAF-Werte zwischen unterschiedlichen Regionen desselben Tumors die Phase der SCNAs relativ zu einer Referenzprobe bestimmt werden (Multi-RegionsReferenz-Phasing, MRP, Abb. 2A, [9]). Der MRP-Algorithmus erlaubt somit die Bestimmung haplotypspezifischer SCNA-Profile und ermöglichte eine sehr viel höhere Genauigkeit in der Rekonstruktion evolutionärer Bäume, da nun beide elterlichen Haplotypen separat modelliert werden können.

\section{Unentdeckte Heterogenität}

In Zusammenarbeit mit Charles Swanton vom Francis Crick Institute in London wendeten wir den neuen MRP-Algorithmus zunächst auf eine Multiregion-LungenkrebsKohorte an. Dort bemerkten wir SCNAs, die in mehreren Regionen eines Tumors vorlagen und ohne Phaseninformation als ein einzelnes Ereignis angesehen worden wären. Tatsächlich handelte es sich jedoch um zwei Ereignisse, die scheinbar unabhängig voneinander auf beiden elterlichen Allelen aufgetreten sind (Abb. 2B). Dieses Phänomen ist nun als gespiegelte subklonale allelische Imbalance (mirrored subclonal allelic imbalance, MSAI) bekannt [10] und wurde später von uns auch in Nierentumoren nachgewiesen [11].

Die MSAI-Ereignisse erwiesen sich für die Suche nach Selektion als besonders relevant, da sie Anzeichen für konvergente Evolution sind $[10,11]$. Wir beobachteten, dass beide SCNAs, die ein MSAI-Ereignis auszeichnen, in ihrem respektiven räumlichen Teil des Tumors oft klonal vorlagen [10]. Dies bedeutet, dass das gleiche SCNA-Ereignis zweimal unabhängig voneinander aufgetreten und anschließend direkt oder indirekt selektiert worden ist - ein deutlicher Hinweis auf kontinuierliche Evolution in der Entwicklung eines Tumors (Abb. 2B).

Um die Häufigkeit dieser MSAI-Ereignisse über Krebstypen hinweg zu untersuchen, begannen wir 2015 mit der Zusammenstellung einer Pankrebs-Kohorte nahezu aller bis dato veröffentlichten Multiregions-Datensätzen. Die Ergebnisse waren erstaunlich: Fast alle untersuchten 22 Krebstypen wiesen in 


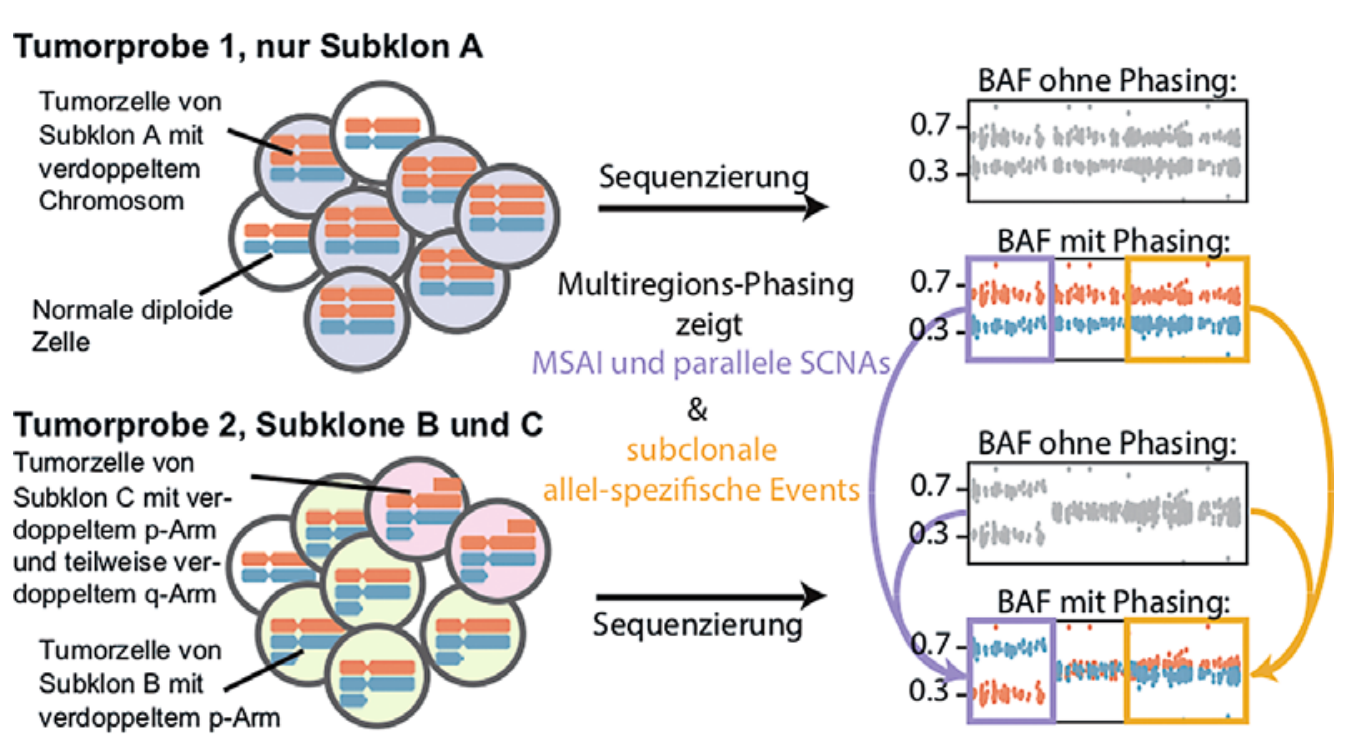

$\triangle$ Abb. 3: MRP findet unentdeckte Heterogenität. Subklonale SCNAs sind oft in Sequenzierdaten schwer zu identifizieren. Eine eindeutig erkennbare allelische Imbalance in einer Region des Tumors (oben) erlaubt es, mittels MRP die Haplotypen zuzuweisen (orange und blau). Wenn in einer anderen Region des Tumors aufgrund von Subklonalität die allelische Imbalance anhand der BAF nicht zu erkennen ist (unten), kann über die Haplotypinformation die Imbalance wieder erkenntlich gemacht werden. Abbildung aus [12].

hohem Maße MSAI-Ereignisse auf. Zudem deckte die Anwendung des MRP-Algorithmus viele hunderte zusätzliche SCNA-Ereignisse auf (Abb. 3), die zuvor aufgrund von Subklonalität oder niedriger Reinheit der Proben nicht erkannt werden konnten [12]. Darüber hinaus gelang es uns mithilfe der Phaseninformation, SCNA-Ereignisse mit hoher Genauigkeit in klonale und subklonale Ereignisse zu unterteilen. Wir konnten so zeigen, dass genomische Regionen mit Onkogenen bevorzugt amplifiziert, und genomische Regionen mit Tumorsupressoren bevorzugt deletiert werden, selbst wenn ausschließlich subklonale SCNA-Ereignisse betrachtet werden - ein weiterer direkter Hinweis auf anhaltende Evolution und Selektion.

Zusammenfassend konnten wir in allen unserer Studien, im Kontrast zur Big BangHypothese, starke Evidenz für die kontinuierliche Evolution von Tumoren, auch nach der initialen malignen Transformation, feststellen.

\section{Literatur}

[1] Nowell PC (1976) The clonal evolution of tumor cell populations. Science 194:23-28

[2] Dagogo-Jack I, Shaw AT (2018) Tumour heterogeneity and resistance to cancer therapies. Nat Rev Clin Oncol 15:8194

[3] Gerlinger M, Rowan AJ, Horswell S et al. (2012) Intratumor heterogeneity and branched evolution revealed by multiregion sequencing. N Engl J Med 366:883-892 [4] Schwarz RF, Trinh A, Sipos B et al. (2014) Phylogenetic quantification of intra-tumour heterogeneity. PLoS Comput Biol 10:e1003535

[5] Schwarz RF, Ng CKY, Cooke SL et al. (2015) Spatial and temporal heterogeneity in high-grade serous ovarian cancer: a phylogenetic analysis. PLoS Med 12:e1001789
[6] Gerstung M, Jolly C, Leshchiner I et al. (2020) The evolutionary history of 2,658 cancers. Nature 578:122-128 [7] Sottoriva A, Kang H, Ma Z et al. (2015) A Big Bang model of human colorectal tumor growth. Nat Genet 47:209-216 [8] Van Loo P, Nordgard SH, Lingjærde OC et al. (2010) Allele-specific copy number analysis of tumors. Proc Natl Acad Sci U S A 107:16910-16915

[9] Schwarz RF, Watkins TBK, Van Loo P (2015) Accurate phasing of allele-specific copy-numbers for inferring tumour evolution with probe-level resolution. ISMB Late Breaking Research. www.iscb.org/cms_addon/multimedia/flvmedia. php?i=2089

[10] Jamal-Hanjani M, Wilson GA, McGranahan N et al. (2017) Tracking the evolution of non-small-cell lung cancer. N Engl J Med 376:2109-2121

[11] Turajlic S, Xu H, Litchfield K et al. (2018) Deterministic evolutionary trajectories influence primary tumor growth: TRACERx Renal. Cell 173:595-610.e11

[12] Watkins TBK, Lim EL, Petkovic M et al. (2020) Pervasive chromosomal instability and karyotype order in tumour evolution. Nature, DOI: 10.1038/s41586-020-2698-6
Funding note: Open Access funding enabled and organized by Projekt DEAL. Open Access: Dieser Artikel wird unter der Creative Commons Namensnennung 4.0 International Lizenzveröffentlicht, welche die Nutzung, Vervielfältigung, Bearbeitung, Verbreitung und Wiedergabe in jeglichem Medium und Forma erlaubt, sofern Sie den/die ursprünglichen Autor(en) und die Quelle ordnungsgemäß nennen, einen Link zur Creative Commons Lizenz beifügen un angeben, ob Änderungen vorgenommen wurden. Die in diesem Artikel 列 genannten Creative Commons Lizenz, sofern sich aus der Abbildungslegen

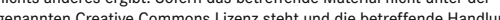

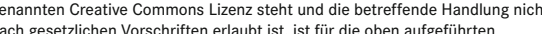
die oben aufgeführten Rechteinhabers einzuholen. Weitere Details zur Lizenz entnehmen Sie bitte der Lizenzinformation auf http://creativecommons.org/licenses/by/4.0/deed.de.

Korrespondenzadresse:

Dr. Roland F. Schwarz

Max-Delbrück-Centrum für Molekulare Medizin (MDC)

Robert-Rössle-Straße 10

D-13125 Berlin

Roland.Schwarz@mdc-berlin.de

Roland F. Schwarz
Informatikstudium. 2009 Promotion an der Universität Würzburg. 2009-2016 Junior
Research Fellow, Wolfson College, University of Cambridge, UK, Postdoc am Cancer
Research UK Cambridge Institute und Marie Curie Fellow am Europäischen Bio-
informatik Institut des EMBL, Hinxton, UK. Seit 2016 Gruppenleiter am Max-Delbrück-
Centrum für Molekulare Medizin, Berlin.

